\title{
Some Dependence Relations of Empirical Belief
}

\author{
JEANETTE MALHERBE \\ University of South Africa
}

Psychological states are not a prima facie natural kind, nor are they susceptible to straightforward empirical observation in the way that material objects are. It is plain though that mental states in general, and empirical beliefs in particular, are complexly related to their physical medium, causes and context, observation of which provides a means of access to them. These facts suggest that empirical beliefs may in some sense be dependent on their physical relata. This paper attempts to set out some of the relations that hold between empirical belief and its determining conditions. First, the idea of a dependence relation is presented. This is followed by the discussion of four such relations of empirical belief. Finally, some consequences are drawn about the nature of empirical belief and justification. A brief statement of seven assumptions about empirical belief which cannot be argued for here, but which make clear the paper's background, is appended.

\section{Relations of determination or dependence}

The idea of a dependence relation has been developed by Kim in the course of his discussion of one particular such relation, supervenience. 1 A starting point for thinking about supervenience, and its historical origin in the literature, is Moore's initial suggestions concerning the supervenience of ethical properties on natural, empirically observable fact. The kind of dependence that the normative features of something have on its natural properties, has since been seen to hold between psychological and physical properties (possibly a special case of normative/natural supervenience), and between macro- and microphysical properties. For instance, a particular empirical belief of S's that the cat is over there, might be said to supervene on a causal array of material fact, including cat, agent, cortex and light; the colour, odour and viscosity of oil might be said to supervene on its microphysical structure.

Supervenience is just one kind of dependence relation; others according to Kim (1984:154), are the determination of an effect by its cause(s) ${ }^{2}$ and the mereological dependence of a whole on its parts. The

1 See Kim 1978, 1982, 1984 especially, 1985 and 1988, especially 399-400; on epistemic supervenience.

2 There are serious difficulties in taking cause/effect as a relation of supervenience, at least if a Moorean conception of supervenience is to be maintained, on which elements in the base set instantiate those in the dependent set. 
notion of dependency, and its converse, determination, is central to the view of the world "not as a mere assemblage of unrelated objects, events, and facts, but as constituting a system, something that shows structure" (Kim, 1984:153), and central also to the intelligibility of that world and the possibility of explanation and understanding.

A dependence relation holds between two different things or kinds of things, in a base and a dependent realm. The presence of individuals in the determining base realm is a necessary condition for that of individuals in its dependent realm (which makes a relation of this sort sensitive to a counterfactual test). A certain configuration of the base set is also sufficient to instantiate an element of the dependent realm. Dependence conditions differ from the usual necessary and sufficient conditions of logic in that a description of the states in the base set is not at the same time a description of states in the dependent set. The two sets however may have the same physical extension. It is possible to say, given certain elements of the base realm and the state dependent on them, that every exact replica of those elements will reproduce the dependent state too, but it is not possible to establish a nomological connection in the other direction and say that a certain dependent state will always be instantiated by base elements, $x, y, z .3$ In Moorean terms, it is impossible that two apples should differ only in this respect, that one is a good apple and the other not good, but goodness is not reducible to a set of natural properties.

We might say, as a working definition of a dependence relation, that if realm $B$, a set of states $b_{1-n}$ with properties BP describable in vocabulary $B V$, is necessary for the instantiation of a realm $D$, where $D=$ [s:s has properties DP describable in DV], then the elements of D depend on those of B. BP and DP, BV and DV, may be completely or not at all coextensive, but cannot, it seems, partially overlap, i.e., there may be dependence relations within one category (e.g., causal dependence among physical events) or across categories (psychophysical dependence), but an appearance of dependence of $D$ partially on $D$ itself and partially on $B$, can usually be resolved by a further reduction of $D$. In what follows, the contention is that empirical belief depends on both physical conditions and other beliefs, but then it must be borne in mind that these other beliefs are themselves dependent on physical conditions, specifically, those states internal to an agent's own neurological system which dispose him to proceed in certain ways and constitute parts of his belief system. I shan't undertake this further reduction here, but will speak of an empirical belief depending in part on other beliefs.

3 Kim however suggests that an accommodating sort of reduction may be possible if we allow a wide enough disjunctive definition of the base realm elements on which a particular supervenience state depends (1984:passim). 
The question then is "Given that any mental state supervenes on a complex physical array, what particular dependence relations, to what particular terms, are discernible in the case of an empirical belief?" There are it seems at least these four things on which any particular empirical belief $B p$ (read "the belief that $p^{\prime \prime}$ ) of an agent $S$ at a time $t$, may variously depend: causal physical conditions at $t$, including $S$ 's afferent nervous system and usually elements external to him, and cortical states of $S$ at $t$ which may be identified as evidential beliefs, general semantic beliefs and probabilistic beliefs relevant to $p$.

2 Dependence relations of empirical beliefs

\subsection{Relevant physical conditions}

Empirical belief arises within the natural order at the instance of physical causes and effects. That is, some sort of cause/effect chain may be picked out from the circumstances of any particular belief, which is essential to it. Notice that both physical causes (usually external to S) and neurophysiological effects are necessary for the generation of an empirical belief; to identify the belief with only the effects is to see it as a selfcontained internal state and this opens the door to a narrow solipsistic materialism. 4 The physical conditions relevant to any empirical belief are rather to be thought of as an array of physical facts definable by virtue of the cause/effect relations among them, upon which the belief depends. A word in qualification of the broad position I am taking: of course there's a sense in which the belief state is internal to $S$ (he can believe falsely and in the absence of his belief state's reference), but the state he's in, Bp, is to be thought of as the effect of which $p$ is, actually or typically, the cause, the idea being that effects define their causes as much as vice versa. In the case of a true empirical belief, content is consonant with the cause/effect array; in the case of a false belief, we must look to the other factors upon which the belief depends (vide infra) besides its cause/effect complex, to understand how content has parted company with its physical matrix.

It is the nature of the afferent nervous system of a rational agent that, when it is brought into causal interaction with some part of its environment, the agent is thereby normally put in a state of readiness to act appropriately with regard to the fact perceived. He is apprised of some way in which the world is. Empirical belief may thus be acquired and held while the sensory mechanisms of the agent are engaged by the (supposed) objects of the belief. The force of the "normally" here is two-fold. Firstly, it

4 Some of the problems attendant on such a view are accounting for the representational content of empirical belief; avoiding scepticism-if a belief depends on nothing outside out heads, we might all very well be brains in vats; and explaining cross-category causation. 
embodies the claim that in its ordinary functioning, without handicapping conditions or blocks on awareness, an afferent nervous system is not just sensitive, but attentive to the environment. 5 It automatically executes the interests of the agent by generating doxastic states which should enable his successful negotiation of his surroundings. (And this is as much as will be said about values as a basis of empirical belief.) Normally then, the agent cannot help but acquire beliefs about the environment, if his senses are stimulated by it. Secondly, the "normally" presupposes a standard rational agent, with the usual neurological and cognitive equipment-some experience and understanding of his world. It does not presuppose that the beliefs acquired in the course of sensory experience are true. Regrettably, it is perfectly normal to get things wrong.

Here is a rough illustration of the physical cause/effect array upon which a true visual belief depends, applicable mutatis mutandis to doxastic states of other perceptual modes. $S$ holds $B p$ at $t$, where $p=$ there is a bend in the road before $S$ at $t$. Bp depends on the cause/effect array of: a road with a bend in it being in a certain spatial relation to $S$ at $t$, a light source adequately illuminating it, an unobstructed visual pathway between the bend and S's eyes, good enough eyes, backed up a proper visual system, focussed on the bend, and a cortex, functioning in a normal, attentive mode.

What of a false belief though-can it depend in any significant sense on a cause/effect array to which the beliefs content does not refer? The answer I think is yes, given that this is a dependence relation of generation and that the belief's content is determined by other factors as well as its cause. For example, $\mathbf{S}$ believes there is a bicycle tire on the lawn before him at $t$, but the array which is the physical matrix of the belief includes besides all the other usual elements, a snake and no tire. Given S's probabilistic belief (vide infra) at $t$, that a snake on his lawn is unthinkable, the physical array is consonant with the belief. Again, S believes there is a bent stick in water when the stick is really straight, because his working semantic beliefs do not include any about refraction. It is thus possible to see how the cause/effect array, coupled with S's other beliefs at $t$, determine even a false current empirical belief.

In the two examples, $S$ did not get things badly wrong. He was mistaken only as to particular properties of the objects in the experience and much of the physical array was still directly correlative with the belief. In a case of grossly disordered experiential belief, as when a hospitalized and hallucinating $S$ believes there are pink rats on the dungeon wall before him, and the belief depends on a pathological brain state induced by toxins, then this particular dependence relation is not so important in

\footnotetext{
5 The homuncular idiom here is harmless; the point could as well be made by saying that the agent is not just sensitive to the environment through the medium of his neuronal system, but attentive to it.
} 
the constitution or to the understanding of the belief, and one must look to S's other beliefs, memories and experiences for the more significant determinants of the belief. I have tried to avoid saying that Bp is caused by its relevant physical conditions, for the reasons mentioned earlier, viz. that this encourages thinking of the belief as identical with only a certain neurological state, but it does seem that this particular dependence relation is one of generation and at least very similar to a causal dependency.

\section{$2.2 \cdot$ Evidential belief}

$S$ may come to have a belief about some way in which the world is (say $\mathrm{p}=$ the chimney is smoking), not through his own experience of it, but indirectly. He may not see the smoking chimney for himself, but learn about it from a credible source, or see a fire in the grate and infer p. Bp may thus be arrived at by $S$ on the basis of other beliefs of his, in putative facts which indicate that $p$ is the case. These beliefs, and what they are about, seem to fall into two main classes.

2.2.1 Belief in the natural evidence for $p: S$ may come to believe that it rained last night $(B p)$ on the basis of his beliefs that the trees are wet $(\mathrm{Bq})$, there are pools on the paths $(\mathrm{Br})$ and the soil is damp (Bs). There are natural relations of cause and effect between $q, r$ and $s$, and $p, s o$ that the presence of the first three is strongly indicative of $p$. If awareness of these relations is an operative part of S's body of semantic or theoretical belief, then, (in the absence of special reasons to the contrary, e.g., S's believing his neighbour's sprinkler was on), if $S$ holds $q, r$ and $s$, he will at the same time accept $p$. An empirical belief that $p$ may thus depend on belief in the direct or natural evidence for $p$, and on the fact that it is such evidence being in S's cognizance. It seems also that there are times when S's acceptance of certain statistics or objective probabilities can act as a kind of evidence for some particular thing's being the case. If $\mathbf{S}$ believes that the worldwide birthrate is 28,000 per day, then in might occur to him at $t$ that, during the last 4 or 5 seconds, a baby has been born on earth-surely a particular empirical belief with respect to time and place.

2.2.2 Belief in testimony for p: $S$ may come to believe that it rained last night as a result of accepting the statement by a trustworthy source that this was so. Here, it is possible to explain the dependence relation in one of two ways. Firstly, there is a Humean sort of view on which S's belief that a source is reliable has the effect of rendering that source's claims of $p$, into something like direct evidence for $p$. The direct natural evidence that $q$ constitutes for $p$, is just the highly regular consequence of $p$ upon $q$; granting a source reliability is allowing that its claims of $p$ are regularly conjoined with the fact that $p$. Hence, to allow that a source is reliable is to give its productions the status of natural evidence. Alternatively, it may be that $\mathrm{Bp}$ is arrived at via acceptance of the truth of 
"p." If $S$ believes that $T$ has uttered "p," and $S$ believes that $T$ is trustworthy, i.e., speaks the truth, then $S$ thereby believes that " $p$ " is true, and so also that $p$ is the case. The utterance is now seen as a conventional rather than a natural sign of the fact that $p$. Either way, $S$ 's belief in testimony that $p$ results in $S$ 's belief that $p$.

As was also the case with the physical cause/effect array, the mere existence of evidential beliefs is normally enough to guarantee that $S$ will hold the dependent belief, and here again the "normally" excludes irrationality and includes the usual background beliefs of cognitive agents. If $S$ sees $a$ fire in the grate and, believing that fire smokes and there are no blocking conditions on this one's smoking, yet rejects the proposition that this fire is smoking, he does so at the price of being irrational. If $T$ asserts that the chimney is smoking, and $S$, believing $T$ credible, yet holds that there is no smoke coming out of the chimney, he is committed to a contradiction. We may think of the physical array and evidential beliefs as the apprising conditions for empirical belief, since these are what inform $S$ of some new state of affairs, or what give him cause to believe that something of which he was not previously cognizant, is the case.

Might there not be many other states which are apprising conditions for empirical belief? Memory, intuition, wishful thinking and brain-washing are some other ways in which it seems $\mathbf{S}$ might come to hold an empirical belief. Memory however, can be ruled out on the grounds that what $S$ remembers is not a fact new to him but something previously believed, and so memory has no apprising force. As for the other three, it is true that $S$ may come by their means to hold that $p$ is the case, in something very like a doxastic state, a state that plays a belief-like role in his functional economy. But these are not the terms of true dependence relations of empirical belief, as those relations were defined in 1 above. $S$ may be in a state of intuiting or wishfully thinking that $p$, without accepting that it is in fact the case and being ready to commit himself to appropriate action; his readiness to act and his conviction (if he does reach one) that the proposition is true, in these two cases seems to depend upon his having forgotten its dubious origins. A conviction that $p$ which is induced in $\mathrm{S}$ by brain-washing hypnotism or the like, is similarly not one in which $\mathrm{S}$ is rationally constrained, simply by virtue of being in that state, to accept $p$. In fact, he would be well advised, and has the reflective capacity, in such circumstances to withhold belief in $p$. S's perception of the fact that $p$, however, either sensorily in a causal physical array, or inferentially, among his existent beliefs, is identical with its acceptance by him.

\section{3}

Semantic belief

There is, however, more needed than just the apprising conditions in order for $\mathbf{S}$ to hold an empirical belief, say, that there are gondolas in Venice. He will need besides some sort of concept or understanding of gondolas and what they are, and some sort of recognition of Venice and 
what it is, and these are cognitive capabilities which involve further beliefs of his about boats, transport, canals, cities and their identity, and ultimately, about things, their properties and relations, their location in space and identity over time, "endless interlocked beliefs. The system of such beliefs identifies a thought by locating it in a logical and epistemic space" (Davidson, 1984:157). This is the familiar Quinean "web of belief," the cognitive context without which a psychophysiological state could not be a belief, since it would have no semantic content.

The term "semantic" should not be taken to imply a necessarily linguistic element in belief. It would be a nominalistic mistake to insist that S have the words "gondola" and "Venice" at his command in order to have the belief that there are gondolas in Venice, although being a linguistic creature, he will recognize his belief under some suitable statement of its content, 6 and will be disposed to assert and assent to such a sentence. "Theoretical" would have done as well, but "semantic" is used here since these are the beliefs that invest the representational content of Bp with its meaning, rather than its existential import, which seems to be a function of the apprising conditions. While both these features of a beliefs representational content--meaning and existential import-are expressed in the same proposition, there is at least this difference between them: that meaning is general while the ontological commitment of a particular empirical belief arises only with the belief. $\mathbf{S}$ may have command of a whole theory of gondolas and Venice and may entertain the thought that there are gondolas in Venice, without having any cause to believe there are any particular gondolas there or anywhere at all.

$S$ does not in any sense infer from his general beliefs about the nature of things, together with his particular experience, to the conclusion that there are gondolas in Venice; rather the reverse. The general beliefs are present in the particular empirical belief and an observer, or $\mathbf{S}$ himself on reflection, is able to say, that since $S$ has a certain particular empirical belief, that he must have certain other general beliefs too. The former is a state of readiness to behave in certain ways, the latter is the capacity to take up that state in the appropriate context. We might explain such a general belief in terms of the disposition to apply a proposition of the form $(x)(F x \rightarrow G x)$, where $F$ represents a quorum of properties perceived by $S$, sufficient for the identification of something as being of a certain kind, and $G$ is a conjunction of all the properties (including $F$ ) which $S$ thinks belong

6 There are difficulties in saying what a "suitable statement" would be of the proposition that there are gondolas in Venice, for the purposes of attributing that belief to $S$. If the sentence that $S$ holds true as a result of seeing gondolas in Venice is "There are odd black boats in the city my uncle lives in," does he believe that there are gondolas in Venice? A better test than the sentence held true might be S's ability to discriminate behaviorally gondolas and Venice from among other boats and cities. 
to things of that kind. For instance, F = brown, matt, odorous, rectangular, sectioned, which is enough for $\mathbf{S}$ to recognize a slab of chocolate by. If it is chocolate, then it is also ( $G=$ ) sweet, nutritious, soft, cocoa-flavoured, yielding to the teeth, soluble, etc., properties which dispose $S$ to proceed in certain ways. If $\mathbf{S}$ did not have the general semantic belief that chocolate was all these things, he could not recognize it, understand what he was looking at, act appropriately towards it, in short, acquire the belief that it was chocolate before him. A New Guinea headhunter with no experience of such stuff might believe it was a very soft wood. Once again, it seems that normally semantic beliefs relevant to the occasion are automatically brought into operation.

Though both particular empirical beliefs and their semantic constitutive beliefs are dispositional states, there is the genuine difference between them referred to above in the phrase "existential import." "Beliefs about particular, spatio-temporally limited, states of affairs have been compared to maps... which, taken together, form one great map of the world...General beliefs are not part of the map. They are dispositions to extend the map according to certain principles" (Armstrong, 1973:99). Talk of this kind should not be read as implying the possibility of some atheoretical kind of given, or of pure data of experience.7 Nevertheless, it seems that a general belief of the kind we are calling "semantic" is operative only in the company of a cause/effect array (and possibly inference), and it is the apprising condition which is essential to S's coming to believe that $p$ is the case, and it is also essential to $S$ 's coming to believe that $\boldsymbol{p}$ is the case, since it is the nature of the experience that determines what general beliefs become operative. If $S$ experiences a gondola on a canal, he is not at liberty to construe it as an acrobat on a tightrope, or does so at the cost of his understanding and effective negotiation of the world.

\subsection{Probabilistic belief}

A probabilistic belief, $\mathrm{Bq}$, is a belief that $\mathrm{S}$ holds at $\mathrm{t}$, antecedently to being apprised of $p$, which renders it more or less likely for him that $p$ is the case. It bears thus on the credibility of the proposition and disposes $S$ to accept or reject it, and to do so with greater or lesser firmness. A probabilistic belief is not necessarily about an objective probability; $q$ may be any sort of proposition, though it must have some sort of perceived bearing for $S$ on the features or context of $p$, and this relationship is such that it can always issue in a formulation, however rough, of the objective probability of $\mathbf{p}$.

Like semantic beliefs, probabilistic beliefs precede S's acquisition of, and are applicable to, the particular Bp. The same beliefs may be both semantic and probabilistic; what distinguishes them as two kinds is that

7 For convincing illustrations of how even immediate perceptual belief is theory laden, see Churchland 1979: 16-21 and 28-34. 
the belief they determine, is determined in different ways by each class. The base realm of semantic beliefs determines how $\mathbf{S}$ will construe an experience and what range of actions he will be disposed to in his acceptance of $p$; the base realms of probabilistic beliefs determines the degree of credibility that $p$ will have for $S$, and (sometimes) which among several possible semantic construals is the most likely. The example above (supra:4) of S's believing there is a tire on his lawn when it is really a snake illustrates how S's background beliefs about the likelihood of $p$ may deflect its credibility for him onto $q$, even when his experience suggests $p$ more strongly than q to him.

An experience's representational content may also appear incredible to $\mathbf{S}$ in the light his beliefs about its or the context's mendacity; tromp l'oeil paintings, wax figures, deliberate deceptions of one sort or another will, if $\mathbf{S}$ is cognizant of the circumstances, cause him to believe not the apparent, but something else. 8 The corollary of this is that when, in normal circumstance, $\mathbf{S}$ believes an ordinary empirical proposition like "There is a cat on the mat," his acceptance of the proposition depends on his having beliefs about the match of context and content, or about context alone, which do not militate against the proposition's credibility. Put into dispositional terms, $S$ is predisposed by his general beliefs that mats are a likely place to find cats, and his particular beliefs that there is nothing untoward about this case, to act as if $p$ were the case when apprised of $p$.

Probabilistic beliefs may act to deflect $S$ from the prima facie belief in a causal array or in the evidence, but they may also promote such belief. $S$ is keen to add a blueeared starling to his bird list, so he goes to a spot where these birds are known to be plentiful and settles near a tree full of their favorite fruits. Presently he sees, somewhat obscured, a bird with a bluish sheen on its head. He believes that there is a blue-eared starling on the branch in front of him. In the presence of his background beliefs about the context of his experience, and the high likelihood of the birds

8 Thus Peacocke (1983:6): "A man may be familiar with a perfect trompe l'oeil violin painted on a door, and be sure from his past experience that it is a trompe l'oeil: nevertheless his experience may continue to represent a violin as hanging on the door in front of him." This should not be read as implying that, for a moment, $\mathrm{S}$ believes here is a violin before him and then remembering his past experience, re-interprets it as a painting; nor I think need it entail that the content of experience is hypothetical in character-that S considers the image of a violin in the light of his other beliefs before deciding what to believe of it. Peacocke's claim is rather that S's visual image of a trompe l'oeil violin is qualitatively indistinguishable from his image of a real violin, which is consistent with my claim that S's immediate experiential belief, depending as it does on image and probabilistic background beliefs, is that there is a painting before him, i.e., he sees the thing as a painting. 
occurring in this context, what would otherwise be too sketchy for thinking "This is a blue-eared starling," becomes sufficient. No wishful thinking need be involved; even for a conscientious and careful believer, if there are contextual factors which increase the probability of $p$, then S's belief that such factors exist automatically increases p's credibility for $S$, or (in the case of an inferential belief) decreases the need for evidence or grounds. Conversely, if $\mathrm{S}$ were to see a blue-eared sitting on his back fence in broad daylight, he would probably not believe his eyes, simply because it is so unlikely a situation to find one of these rare birds in.

So much for immediate belief. Here is a comparative example of how inferential belief depends on probabilistic beliefs. $\mathbf{S}$ goes into his garden in the early morning and sees that the shrubs are wet, there are pools on the paths and the soil is damp. If it is in December, $\mathbf{S}$ concludes that it rained in the night; if it is in June, $S$ thinks that his neighbour's sprinkler has been on. The first belief depends on S's further beliefs about the likelihood of rain being high in December; the second, on his beliefs about rain being almost unheard of in June. This example illustrates too that $\mathrm{Bp}$ is not typically arrived at by S's inferring it from premisses of probabilistic beliefs plus experiential or evidential beliefs. That the experience or evidence assumes one particular character for $S$, rather than another, is explicable in the light of his background beliefs.

There are (at least) two possible objections to regarding these probabilistic beliefs as dependence conditions for empirical belief. Firstly, does empirical belief essentially depend upon such beliefs? Aren't they rather an adjunct to a particular empirical belief, perhaps acting in the exceptional case to bar or admit belief, but normally playing a merely cautionary or confirmatory role? A probabilistic belief disposes $S$ to accept or reject $\mathrm{p}$ with greater or lesser firmmess (supra:8). Surely the implication of this, in conjunction with the thesis of apprising conditions in $\mathbf{2 . 1}$ and 2.2 above, is that probabilistic beliefs are not essential to the acceptance of an empirical fact. If this objection can be answered, and it can be shown that they are necessary, then a second question may be raised: are probabilistic beliefs in any real sense different from the particular empirical belief they "determine," or should they not rather be regarded as identical with it? The degree of $p$ 's subjective probability for $S$ at $t$ is surely just the more or less firmly held belief that $p$ is the case. And if this is so, there can once again, though for a different reason, be not talk of dependence of the one on the other. The first objection would have it that probabilistic beliefs are only contingently related to an empirical belief, the second, that they are in a relation of identity; either way, the relation is not one of dependence.

I lack space here to deal fully with these objections, but think that both can be defeated. Firstly, that p's likelihood in terms of S's other relevant empirical beliefs, is necessary for $\mathrm{Bp}$, and that p's acceptance after an initial rejection depends on a revision of those other beliefs, may 
be established both by exemplification and an argument on coherentist lines which equates credibility with acceptability, with the consistency of $p$ with $S$ 's whole doxastic set. Secondly, it may be granted that p's subjective probability for $\mathbf{S}$ at $\boldsymbol{t}$ is nothing but the sum of his other beliefs which bear on p's likelihood, so long as we remember that this is not all there is to a particular empirical belief. There are also the apprising and semantic conditions, so that Bp cannot be completely identified with the probabilistic beliefs it depends upon. That they are an integral part of Bp is not a difficulty for, but a vindication of, the dependency thesis.

\section{Conclusions}

I have said almost nothing about a different kind of dependence relation of empirical beliefs-their occurring at the instance of the agent's interests, needs, values. Since a belief is a means-choice disposition, it is integrally related to the agent's purposes, and he acquires beliefs about things only as he conceives them in functional interaction with himself. 9 However, it is incumbent on agents to discount their immediate local interests in forming empirical beliefs. Given that absolute truth is a value to which all empirical belief aspires and that all normal rational agents are in consequence type-identical in their relations with the material world, the needs and interests upon which empirical beliefs essentially depend will be the same across the board. The teleological relations of empirical belief may thus have less, or a different kind of, determinative power than the four discussed above. Still, values represent a base realm which would require investigation in any full study of the dependence relations of empirical belief. There may be others.

Returning to the four conditions set out above, it is fairly safe to say that any particular empirical belief is determined in part by apprising conditions, by semantic and by probabilistic beliefs of the agent. The first gives $S$ reason to think that $p$ is the case; the second gives $p$ a cognitive content that $S$ can grasp; the third gives $p$ acceptability in terms of S's other empirical beliefs.

This thesis has various applications. What, on a purely propositional view of belief, would be the same belief (i.e., belief in the one proposition) may depend to different degrees on these three terms, in different agents or the same agent at different times. For instance, agents $\mathbf{Q} \mathbf{R}$ and $\mathbf{S}$ all believe that there are dugongs in the Sargasso. $\mathbf{Q}$ has encountered them, though he does not have their or their location's name; $\mathbf{R}$ has forgotten how he came by the fact and has only the sketchiest idea of what dugongs or the Sargasso are, but p's intersubjective assertibility has confirmed him in Bp; $S$ extrapolates on evolutionary principles from

9 For a definition of belief as essentially the choice of a means to satisfy an individual's "ensemble of ends," see Goldstick (1989:passim). 
what he knows of marine animals and their habitats to Bp. It seems reasonable to say that the physical array of which $Q$ is a part is centrally determinative of Q's Bp (belief de re); that whatever substance R's Bp has is derived from R's semantic beliefs (de dicto); and that $S^{\prime} s \mathrm{Bp}$ is a hypothesis that depends in the main probabilistically on other beliefs of $S$. How these three instances of the "same" belief differ is thus explicable with reference to the different dependence relations of each. It is still the case that all three terms (apprising conditions, semantic and probabilistic beliefs) must be present in each belief.

There are also implications in this dependency thesis for empirical justification, in particular for the traditional standoff between coherentists and foundationalists. If the physical cause/effect array is indeed a determining factor of some empirical beliefs, then there is at least one important respect in which they are not dependent on the existing beliefs of $S$ at $t$, and in which it might therefore be argued their justification cannot consist in relations of coherence. In the case of inferential beliefs too, it seems that their epistemic status, and so perhaps their justification too, may consist in relations of at least three different kinds.

\section{APPENDIX-SEVEN ASSUMPTIONS ABOUT EMPIRICAL BELIEF}

1. Empirical belief is belief about some way in which the world is. As such, it has a guaranteed extension, since it is correct to say in any particular case that, of spacetime region $x y, S$ believes $p$. Empirical belief is not of course guaranteed true; what $S$ believes of $x y$ may always be false. 2 A single empirical belief s content is specifiable in a proposition which is also a statement of (supposed) material fact and which is made true by the obtaining of that fact. The focus in this paper is on the doxastic state of an individual agent, and an egocentric view of a "fact" will therefore be taken, from which, talk of putative or supposed facts is natural.

3. Toothaches (and somatic sensations in general) do not represent the world as being a certain way to $S$, but are the immediate experience of his own psychophysiological states and are thus not empirical doxastic states, though $\mathbf{S}$ may infer from such an experience the empirical belief that his tooth is decayed. A hallucination that there are rats on the floor, while it also is a experience of nothing outside S's own neurological system, is nevertheless empirical since its reference is "some way in which the world is."

4. The assumption will be that there is more to a belief than a positive, negative or neutral attitude to a proposition; there is also (at least) its character, including, in the case of empirical belief, sensory and 
indexical elements, and sometimes qualia too; its setting in the unique doxastic set of each agent, which partially determines what sort of content the belief has; and its causal history-an empirical belief is acquired it seems through (varying degrees of the instrumental agency of the sensory mechanisms. In countering the rooted inclination to take a belief as only $+p,-p$, or ? $p$, it helps to think of Bp as "the doxastic psychological state that p." A corollary of this view of belief, is that experience is essentially doxastic in character.

5. The underlying philosophy of mind here is materialistic, weakly non-reductive. The intentional idiom is useful for its convenience and to make up for our neurophysiological ignorance, but beliefs, especially empirical beliefs, and their contents, could in principle be completely understood in extensional terms. Psychophysiological naivete is responsible for the seeming anomalousness of the mental. A single grossly defined extensional array might serve as the base of many intensionally specified beliefs, but to each such belief there corresponds a unique fully defined physical base in which cortical states are fully particularized. If a cortical state is a belief state, it disposes $\mathbf{S}$ to behave in a certain way. A belief is thus a state which arises within the physical onder at the instance of natural causes and effects.

6. It is the dependence relations of a single empirical belief of an agent $S$ at a time $t$, rather than his belief about the world in general that will be explicated, with this proviso, that the idea of an individual belief is a convenient artifice, conclusions about which should be generalized to the undifferentiated empirical belief of the agent. This seems advisable in the light of the fact that the complex psychological state which includes S's expectations and his current and remembered experience, i.e., his doxastic state vis a vis the world, if exhaustively specified in terms of single belief states at $t$, would yield an enormous, though not I think infinite, number of beliefs.

7. There is a serious, epistemic kind of believing in which a belief, albeit a subjective probability, is not conditional in character or a hypothesis to be tested in action, but a proposition held with some. conviction, to whose truth $S$ is committed.

\section{REFERENCES}

Armstrong, D. M. 1973. Belief, truth and knowledge. Cambridge: Cambridge University Press.

Churchland, P. M. 1979. Scientific realism and the plasticity of mind. Cambridge: Cambridge University Press.

Davidson, D. 1984. Enquiries into truth and interpretation. Oxford: Oxford University Press. 
Goldstick, D. 1989. "Belief," in American Philosophical Quarlerly, 26, 3 J1 89, 231-238.

Kim, J. 1978. "Supervenience and nomological incommensurables," in APQ, 15, 2, Ap 78, 149-156.

1982. "Psychophysical supervenience," in Phil Studs, 41, Ja 82, 5170.

1984. "Concepts of supervenience," in Phil Phen Res, LXV, 2, De 84, 153-176.

1985. "Psychophysical laws," 369-386 in Actions and events. Perspectives on the philosophy of Donald Davidson, eds. E. LePore \& B. McLaughlin. Oxford: Basil Blackwell.

-1988. "What is naturalized epistemology?," in Philosophical Perspectives, 2, Epistemology, 381-405.

Peacocke, C. 1983. Sense and content. Experience, thought and their relations. Oxford: Clarendon Press. 